\title{
A CLASS OF GEOMETRIC LATTICES
}

\author{
K. ROGERS AND E. G. STRAUS
}

Communicated by E. F. Beckenbach, December 26, 1959

1. Introduction. By an $n$-dimensional lattice $\Lambda$ we mean, as usual, an additive subgroup with $n$ linearly independent generators of the vectors in Euclidean $n$-space, $R^{n}$. If we denote by $Z^{n}$ the lattice of vectors with integral components, then $\Lambda$ is the image of $Z^{n}$ under a nonsingular linear transformation:

$$
\Lambda=\left\{A \boldsymbol{u} \mid \boldsymbol{u} \in Z^{n}\right\}, \quad \operatorname{det} A \neq 0 .
$$

The matrices mapping $Z^{n}$ onto $\Lambda$ constitute a coset $A U$ of the subgroup of all integral unimodular matrices, and so $\operatorname{det} \Lambda=|\operatorname{det} A|$ is well-defined. It is convenient to use the same name $\Lambda$ for the pointlattice of all points $P$ such that $O P$ is in $\Lambda$.

Minkowski [2] showed that every lattice of determinant one contains a point other than the origin 0 in the cube

$$
\left\{\left(x_{1}, \cdots, x_{n}\right)|| x_{i} \mid \leqq 1, i=1, \cdots, n\right\},
$$

and that the same holds if any $n-1$ of the signs are replaced by strict inequality. Those unimodular lattices, such as $Z^{n}$, which have only the origin in common with the open cube shall be called critical, as shall the corresponding matrices. Minkowski conjectured, and Hajos [1] proved in 1938, that a critical lattice must contain one of the points $\left(\delta_{i 1}, \cdots, \delta_{i n}\right), i=1, \cdots, n$. If $A$ is critical then so is any matrix obtained from it by permuting rows and post-multiplication by integral unimodular matrices: such matrices will be called equivalent to $A$. An induction argument shows that Hajos' theorem is the same as the assertion:

$A$ is critical if and only if it is equivalent to a matrix with ones on the diagonal and all zeros above.

Siegel [3] tried to prove Minkowski's conjecture by showing that, if $A$ is critical, then each point other than 0 of the lattice corresponding to $A$ has at least one coordinate in $Z^{*}$, the set of nonzero integers. If we consider the set of matrices $A$ defined by the property

$$
u \in Z^{n}, \quad u \neq 0 \Rightarrow A u \text { has a component in } Z^{*},
$$

then Hajos' theorem would follow from Siegel's result, if it were true that every $A$ with property $(\mathrm{P})$ has an integral row. For in that case we could prove by induction on $n$ that $A$ is equivalent to a triangular matrix with zeros above the diagonal and positive integers on the 
diagonal. Thus if $|\operatorname{det} A|=1$, then the diagonal elements must be 1 .

Conversely, since every $A$ with property (P) and $|\operatorname{det} A|=1$ is clearly critical, Hajos' theorem shows that the combination of $(\mathrm{P})$ with $|\operatorname{det} A|=1$ does imply that $A$ has an integral row.

Unfortunately property (P) alone does not suffice for $n \geqq 5$ as is shown by the example

$$
A=\left(\begin{array}{ccccc}
1 / 3 & 0 & 0 & 0 & 0 \\
0 & 1 / 3 & 0 & 0 & 0 \\
1 / 2 & 3 / 2 & 3 & 0 & 0 \\
1 & 3 / 2 & 0 & 3 & 0 \\
3 / 2 & 1 & 0 & 0 & 3
\end{array}\right) .
$$

Here $A$ has property (P) but no row is integral. However we were able to show that property $(\mathrm{P})$ does imply that $\operatorname{det} A \in Z^{*}$ and to obtain various generalizations of that result.

2. The special case of the main theorem enunciated at the end of the introduction, when $A$ is rational, is included in:

TheOREM I. Let $K$ be an algebraic number field of class-number one, and let $J$ be its ring of integers. If an n-by-n matrix $A$ over $K$ has the property

$$
u \in J^{n}, \quad u \neq 0 \Rightarrow A u \text { has a component in } J^{*},
$$

then $\operatorname{det} A \in J^{*}$.

Proof. If $A$ has property (P) then $\operatorname{det} A \neq 0$, so we only have to show that $\operatorname{det} A \in J$. The theorem being trivial for $n=1$, we use induction. Take an $A$ with (P) and assume that $\operatorname{det} A$ is not in $J$. We shall deduce the existence of an $A$ with (P) such that $\operatorname{det} A=1 / q$, where $q$ is a prime of $J$; and then a contradiction follows from a pseudo-analogue of Minkowski's theorem, which we shall prove.

Lemma 1. If there is an $A$ with (P) such that $\operatorname{det} A$ is not in $J$, then there exists $A_{1}$ with $(\mathrm{P})$ such that $\operatorname{det} A_{1}=a / q$, where $a \in J$ and $q$ is a prime of $J$ which does not divide a.

Proof. Since $J$ has unique factorization into primes and since $\operatorname{det} A$ is not integral, we have $\operatorname{det} A=a / q b$, where $a, b, q$ are in $J, q$ is a prime element, and $(a, q b)=J$. Multiplying the first row of $A$ by $b$ gives a matrix $A_{1}$ with $(\mathrm{P})$ such that $\operatorname{det} A_{1}=a / q$, as required.

It is easy to remove from each row of $A$ any common denominator prime to $q$ without affecting $(\mathrm{P})$ or the fact that the determinant has 
denominator $q$. Thus we may assume that: (i) $A$ has property (P), (ii) $\operatorname{det} A=a / q$, where $q$ is a prime not dividing $a$, (iii) some power of $q$ is a denominator for $A$.

Lemma 2. If there is an $A$ with properties (i), (ii) and (iii), then there exists an $A$ with (P) such that $\operatorname{det} A=1 / q$.

Proof. Since $J$ has only principal ideals, we can find an integral unimodular matrix $U$ such that $A U$ is triangular. By the properties of $A$ we may write the diagonal elements of the new $A$ as $a_{i i}=a_{i} q^{b_{i}}$, $a=\prod a_{i}, \sum b_{i}=-1$. Multiplying a column or a row of $A$ by a nonzero integer does not affect (P). Hence, if $A^{\prime}$ is obtained from $A$ by multiplying the first $n-1$ columns by $a_{n}$, then $A^{\prime}$ has $(\mathrm{P})$ and only powers of $q$ occur in the denominators in $A^{\prime}$. Since $a_{n}$ is prime to $q$, we get a matrix $A^{\prime \prime}$ with $(\mathrm{P})$ when we divide each row of $A^{\prime}$ by $a_{n}$. The net result is that $a_{i j}^{\prime \prime}=a_{i j}$ if $(i, j) \neq(n, n), a_{n n}^{\prime \prime}=q^{b_{n}}$. Similarly we can multiply the first $n-2$ columns by $a_{n-1}$ and then divide the first $n-1$ rows by $a_{n-1}$, and so on. We end up with the matrix

$$
A^{*}=\left(\begin{array}{lllll}
q^{b_{1}} & 0 & \cdots & 0 \\
a_{21} & q^{b_{2}} & 0 & \cdots & 0 \\
a_{2} a_{31} & & \cdot & & \\
\vdots & & & \cdot & \\
a_{2} & \cdots & a_{n-1} a_{n 1}, & \cdots & q^{b_{n}}
\end{array}\right)
$$

which has property (P) and determinant $q^{\Sigma b_{i}}=q^{-1}$, as required.

Since $\mid$ norm $q^{-1} \mid<1$, the desired contradiction follows from the next theorem.

THEOREM II. If $A$ is an $n$-by-n matrix over $K$ such that $0<|\operatorname{norm}(\operatorname{det} A)|<1$, then there exists $u \neq 0$ in $J^{n}$ such that $A u$ has no component in $J^{*}$.

Proof. Let $d_{i}$ be a common denominator for the elements of the $i$ th row of $A$, and let $A_{1}$ denote the matrix obtained by multiplying each row of $A$ by its $d_{i}$. Then

$$
\begin{aligned}
0<\left|\operatorname{norm}\left(\operatorname{det} A_{1}\right)\right| & =\left|\prod_{i} \operatorname{norm} d_{i} \cdot \operatorname{norm}(\operatorname{det} A)\right| \\
& <\left|\prod_{i} \operatorname{norm} d_{i}\right| .
\end{aligned}
$$

Hence it is sufficient to prove: 
LEMmA 3. If $B$ is an $n$-by-n matrix over $J$ such that $0<|\operatorname{norm}(\operatorname{det} B)|$ $<\prod$ norm $d_{i}$, where the $d_{i} \in J$, then there exists $a u \neq 0$ in $J^{n}$ such that $B u=v$, where $v_{i}=0$ or $d_{i} \nmid v_{i}$, for each $i$.

Proof. Rado [4] has given a proof of Minkowski's theorem on linear forms which can easily be generalised to prove this lemma. We may assume $B$ has been put in the form of a triangular matrix, with zeros above the diagonal and elements $a_{1}, \cdots, a_{n}$ on the diagonal. Hence $0<\mid \prod_{i=1}^{n}$ norm $a_{i}|<| \prod$ norm $d_{i} \mid$. Let $\alpha_{i}, \delta_{i}$ run over complete sets of residues mod $a_{i}$ and mod $d_{i}$, respectively. Then the number of vectors $\alpha$ is $\mid \Pi$ norm $a_{i} \mid$, and the number of $\delta$ is $\mid \prod$ norm $d_{i} \mid$. Thus, there are more vectors $\delta$ than $\boldsymbol{\alpha}$. Now we assert that for given $\delta$ there is one and only one $\alpha$ such that the equation

$$
B u=\delta+\alpha
$$

is solvable for $\boldsymbol{u} \in J^{n}$. For $n=1$, the equation is $a_{1} u=\delta_{1}+\alpha_{1}$; and for given $\delta_{1}$ this is solvable with integral $u$ if and only if $\alpha_{1}$ is in a certain residue class $\bmod a_{1}$, hence for one and only one $\alpha_{1}$. Assuming the assertion true for $n-1$, we know that the first $n-1$ equations are solvable, for given $\delta_{1}, \cdots, \delta_{n-1}$, with integral $u_{1}, \cdots, u_{n-1}$ for one and only one $\left(\alpha_{1}, \cdots, \alpha_{n-1}\right)$. Finally, for given $\delta_{n}$, the equation

$$
\left(b_{n 1} u_{1}+\cdots+b_{n^{\prime} n-1} u_{n-1}\right)+a_{n} u_{n}=\delta_{n}+\alpha_{n}
$$

is solvable with integral $u_{n}$ for one and only one $\alpha_{n}$, as in the case $n=1$.

Since there are more $\delta$ than $\alpha$, we can find distinct $\delta, \delta^{\prime}$ and some $\boldsymbol{\alpha}$ such that $B \boldsymbol{u}=\boldsymbol{\delta}+\boldsymbol{\alpha}$ and $B \boldsymbol{u}^{\prime}=\boldsymbol{\delta}^{\prime}+\boldsymbol{\alpha}$, where $\boldsymbol{u}$ and $\boldsymbol{u}^{\prime}$ are in $J^{n}$. Since $\delta \neq \boldsymbol{\delta}^{\prime}$, therefore $\boldsymbol{u}-\boldsymbol{u}^{\prime} \neq \mathbf{0}$ and $B\left(u-\boldsymbol{u}^{\prime}\right)=\boldsymbol{\delta}-\boldsymbol{\delta}^{\prime}$. Now $\delta_{i}, \delta_{i}^{\prime}$ are either equal or in distinct residue classes $\bmod d_{i}$; hence $\delta_{i}-\delta_{i}^{\prime}$ is either zero or indivisible by $d_{i}$. This proves the lemma, with $\boldsymbol{v}=\mathbf{\delta}-\boldsymbol{\delta}^{\prime}$, and also completes the proofs of Theorems I and II.

3. Generalizations. Theorem I holds without the restriction that the elements of $A$ lie in $K$.

Lemma 4. Let $K$ be any field with at least $n$ elements, and let $A$ be an $n$-by-n matrix over some $K$-module, such that

$$
u \in K^{n}, \quad u \neq 0 \Rightarrow A u \text { has a component in } K^{*} .
$$

Then some row of $A$ consists of elements of $K$, not all zero, and $A$ is equivalent, under interchange of rows and right-multiplication by a nonsingular matrix over $K$, to a triangular matrix whose diagonal elements are in $K^{*}$. 
Proof. Since the lemma is true for $n=1$, take $n \geqq 2$. If we write $L_{i}=\left\{u \mid u \in K^{n},(A u)_{i} \in K\right\}$, then $L_{i}$ is a subspace of $K^{n}$, and $L_{i}=K^{n}$ if the $i$ th row of $A$ is zero, an a priori possibility. Condition $\left(\mathrm{P}^{\prime}\right)_{n}$ shows that $K^{n}$ is the union of the $L_{i}$ which correspond to a nonzero row of $A$. If one of these $L_{i}=K^{n}$, then the $i$ th row of $A$ is nonzero and all its elements are in $K$. Otherwise, we must have $K^{n}$ equal to a union of at most $n$ proper subspaces. We now show that this is impossible when $\#(K) \geqq n$. Suppose we have reduced down to the case $K^{n}=L_{1} \cup L_{2} \cdots \cup L_{m}$, with $m \leqq n$ and minimal. Hence there exist $\boldsymbol{u}, \boldsymbol{v}$ in $K^{n}$ such that $\boldsymbol{u}$ is not in $L_{2} \cup L_{3} \ldots \cup L_{n}$ and $\boldsymbol{v}$ is not in $L_{1}$. By intersecting each side of the above equation with the plane $K u+K v$, we find that the plane equals the union of at most $n$ lines through the origin. This is clearly false if $K$ is an infinite field; and in the case $q=\#(K)$, the total number of points would be $q^{2}=m(q-1)$ +1 , hence $m=q+1$, in contradiction to $q \geqq n \geqq m$.

We can now switch the row whose elements are in $K$ to the first row and then by linear combinations of columns with coefficients from $K$ reduce $A$ to the form

$$
\left(\begin{array}{lc}
a_{1} & 0 \cdots \\
\cdot & B
\end{array}\right)
$$

where $a_{1} \in K^{*}$. Since $B$ has property $\left(\mathrm{P}^{\prime}\right)_{n-1}$ we can now proceed by induction to prove the last part of the lemma.

CoROLlary. If the field $K$ in Lemma 4 is an algebraic number field of class-number one, then property $\left(\mathrm{P}^{\prime}\right)$ implies the equivalence of $A$ to a triangular matrix with diagonal elements in $K^{*}$ by switching of rows and right-multiplication by a unimodular matrix over $J$, the integers of $K$.

Proof. We again switch the row with elements in $K$ to the first row. Since the class-number is one, we can form a linear combination of the columns with coefficients in $J$ to give a new first column such that in the new matrix $a_{11}$ divides all terms in the first row, i.e. $a_{1 i} \in a_{11} J, i=1,2, \cdots, n$. Then by subtracting integral multiples of the first column from other columns we can reduce $a_{1 i}$ to 0 for $i=2, \cdots, n$. The induction proceeds as before.

Lemma 5. If $K$ is the field of Theorem I, $A$ an n-by-n matrix over some $K$-module, $K_{1}$, and $A$ has property $(\mathrm{P})$, then there is a matrix $A^{\prime}$ over $K$ with property $(\mathrm{P})$ such that $\operatorname{det} A=\operatorname{det} A^{\prime}$.

Proof. Since $(\mathrm{P})$ implies $\left(\mathrm{P}^{\prime}\right)$, we can triangularize $A$, as in the 
corollary. We can regard $A$ as being over the $K$-module obtained by adjoining the $a_{i j}$ to $K$, say $K^{\prime}$. If $1, \xi_{1}, \cdots, \xi_{N}$ be a basis for $K^{\prime}$ over $K$, then

$$
A=A^{\prime}+A_{1} \xi_{1}+\cdots+A_{N} \xi_{N},
$$

say, where $A^{\prime}$ and the $A_{i}$ are over $K$, all are triangular, the $A_{i}$ having all zeros on the diagonal, while $A^{\prime}$ coincides with $A$ on the diagonal. Hence $\operatorname{det} A=\operatorname{det} A^{\prime}$. Finally, since

$$
A u=A^{\prime} u+\sum_{i=1}^{N} \xi_{i}\left(A_{i} u\right)
$$

it is clear that $A^{\prime}$ also has property (P).

We have thus proved the desired generalization:

Theorem I'. Theorem I remains valid under the hypothesis that the matrix $A$ is over some $K$-module.

In particular, if $A$ is over the reals and transforms every nonzero integer vector into a vector with at least one component in $Z^{*}$, then $\operatorname{det} A$ is in $Z^{*}$.

\section{REFERENCES}

1. G. Hajos, Über einfache und mehrfache Bedeckung des n-dimensionalen Raumes mit einem Würfelgitter, Math. Z., vol. 47 (1941) pp. 427-467.

2. H. Minkowski, Geometrie der Zahlen, Leipzig, Teubner, 1910, §30, p. 72.

3. C. L. Siegel, Neuer Beweis des Satzes von Minkowski über lineare Formen, Math. Ann. vol. 87 (1922) pp. 36-38.

4. R. Rado, A proof of Minkowski's theorem on homogeneous linear forms, J. London Math. Soc. vol. 9 (1934) pp. 164-165.

University of California, Los Angeles 